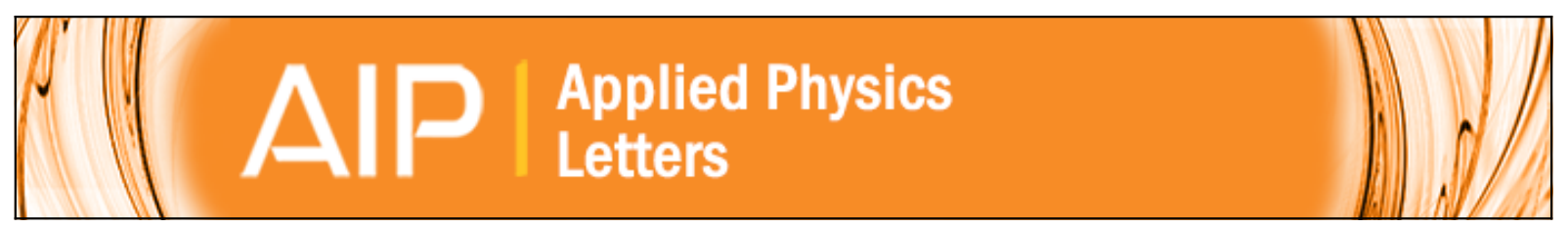

\title{
Superscattering of light optimized by a genetic algorithm
}

Ali Mirzaei, Andrey E. Miroshnichenko, llya V. Shadrivov, and Yuri S. Kivshar

Citation: Applied Physics Letters 105, 011109 (2014); doi: 10.1063/1.4887475

View online: http://dx.doi.org/10.1063/1.4887475

View Table of Contents: http://scitation.aip.org/content/aip/journal/apl/105/1?ver=pdfcov

Published by the AIP Publishing

\section{Articles you may be interested in}

Nanoantennas for nanowire photovoltaics

Appl. Phys. Lett. 105, 113107 (2014); 10.1063/1.4896109

Numerical investigation of optical Tamm states in two-dimensional hybrid plasmonic-photonic crystal nanobeams J. Appl. Phys. 116, 043106 (2014); 10.1063/1.4891222

Hybrid plasmonic waveguide in a metal $\mathrm{V}$-groove

AIP Advances 4, 017103 (2014); 10.1063/1.4861582

Chiroptical hot spots in twisted nanowire plasmonic oscillators

Appl. Phys. Lett. 102, 043103 (2013); 10.1063/1.4789529

Optimal design of nanoplasmonic materials using genetic algorithms as a multiparameter optimization tool

J. Chem. Phys. 129, 064706 (2008); 10.1063/1.2961011

\section{High-Voltage Amplifiers}

-Voltage Range from $\pm 50 \mathrm{~V}$ to $\pm 60 \mathrm{kV}$

- Current to 25A

Electrostatic Voltmeters

- Contacting \& Non-contacting

- Sensitive to $1 \mathrm{mV}$

- Measure to $20 \mathrm{kV}$
ENABLING RESEARCH AND

INNOVATION IN DIELECTRICS,

ELECTROSTATICS, MATERIALS, PLASMAS AND PIEZOS

TRek www.trekinc.com 


\title{
Superscattering of light optimized by a genetic algorithm
}

\author{
Ali Mirzaei, ${ }^{\text {a) }}$ Andrey E. Miroshnichenko, llya V. Shadrivov, and Yuri S. Kivshar \\ Nonlinear Physics Center, Research School of Physics and Engineering, Australian National University, \\ Canberra ACT 0200, Australia
}

(Received 20 May 2014; accepted 12 June 2014; published online 8 July 2014)

\begin{abstract}
We analyse scattering of light from multi-layer plasmonic nanowires and employ a genetic algorithm for optimizing the scattering cross section. We apply the mode-expansion method using experimental data for material parameters to demonstrate that our genetic algorithm allows designing realistic core-shell nanostructures with the superscattering effect achieved at any desired wavelength. This approach can be employed for optimizing both superscattering and cloaking at different wavelengths in the visible spectral range. (C) 2014 AIP Publishing LLC.
\end{abstract}

[http://dx.doi.org/10.1063/1.4887475]

Subwavelength multi-layer structures may demonstrate very unusual optical properties which can be employed for engineering functional metadevices. ${ }^{1}$ In particular, it was shown that multi-shell plasmonic nanoparticles may possess highly unusual scattering characteristics related to the reduced scattering attributed to invisibility cloaking and scattering cancellation. ${ }^{2-4}$ In addition to cloaking, it was shown that multi-layer nanoparticles can exhibit an enhanced scattering or subwavelength superscattering, ${ }^{5}$ when the scattering cross-section of a subwavelength structure exceeds substantially its geometrical cross-section and can be made very large. This effect can be very important for practical applications in photovoltaics and solar cells, sensing and biomedicine. $^{6-11}$

Superscattering of light can be achieved by engineering an overlap of resonances of different modes at the same wavelength, ${ }^{12,13}$ and this effect is very sensitive to the material parameters being drastically suppressed by material losses. Recently, we have studied both cloaking and superscattering for multi-shell nanowires, and suggested a simple design of nanoplasmonic structures which can demonstrate both superscattering and cloaking effects in the visible frequency range under experimentally realistic parameters. ${ }^{14} \mathrm{~A}$ design and optimization of such systems remain a real challenge, so the big question is how to engineer a nanoscale structure to achieve the superscattering effect at any desired wavelength.

In this Letter, we employ a genetic algorithm (GA) for optimizing the scattering cross section of a multi-core plasmonic nanowire and demonstrate that the superscattering can be realized at any desired wavelength, for realistic material parameters. We also suggest that this approach can be employed for optimizing both superscattering and cloaking at different wavelengths in the visible spectrum.

To demonstrate a possibility to construct a subwavelength superscatterer, we choose a cylindrical multilayer structure. In Fig. 1, we show a general schematics of the problem. The incident plane wave propagates along the $x$ axis with the magnetic field polarised parallel to the cylinder axis, $\mathbf{H}^{i n c}=\hat{\mathbf{a}}_{z} H_{0} \exp \left(-i \omega t+i k_{0} r \cos (\varphi)\right)$, where $H_{0}$ is

${ }^{a)}$ Electronic mail: ali.mirzaei@anu.edu.au the amplitude of the incident magnetic field. For an $L$-layer structure, which is embedded in a free space, we represent the field in each layer in the form $\mathbf{H}_{z}(r, \varphi)=\hat{\mathbf{a}}_{z} H_{0} \sum_{n=0}^{+\infty}$ $N(n) H_{z}^{n}(r) \cos (n \varphi)$, where $H_{z}^{n}(r)=i^{n}\left[\tau_{l}^{n} J_{n}(\bar{r})+\rho_{l}^{n} H_{n}^{(1)}(\bar{r})\right]$ and $\mathbf{E}_{\varphi}(r, \varphi)=\hat{\mathbf{a}}_{\varphi} E_{0} \sum_{n=0}^{+\infty} N(n) E_{\varphi}^{n}(r) \cos (n \varphi)$ with $E_{\varphi}^{n}(r)$ $=i^{(n+1)} \varepsilon(r, \lambda)^{-1 / 2}\left[\tau_{l}^{n} J_{n}^{\prime}(\bar{r})+\rho_{l}^{n} H_{n}^{(1) \prime}(\bar{r})\right]$, where $\bar{r}=r k(r)$ is a local size parameter, $k(r)=k_{0} \sqrt{\varepsilon(r, \lambda)}$ is the wavenumber, $J_{n}$ and $H_{n}^{(1)}$ are the $n$-th order Bessel and Hankel functions of the first kind, respectively; $n$ is the mode number, $r$ is the radial coordinate within the $l$-th layer, $\varepsilon(r, \lambda)$ is the dielectric constant at the corresponding point in space for a given wavelength, $\tau_{l}^{n}$ and $\rho_{l}^{n}$ are the $n$-th mode coefficients in the $l$-th layer which have to be found by solving the boundary condition equations for the tangential components of the fields, $H_{z}$ and $E_{\varphi} \cdot{ }^{15,16}$ Additionally, we put $\rho_{1}^{n}=0$ to avoid singularity of Hankel functions at the origin, and $\tau_{L+1}^{n}=1$ for each $n$, so that our equations also describe the incident plane wave as a superposition of cylindrical modes. Coefficient $N(n)=1$ for $n=0$ and for all other modes $N(n)=2$ due to the symmetric relation of the Bessel functions with positive and negative indices.

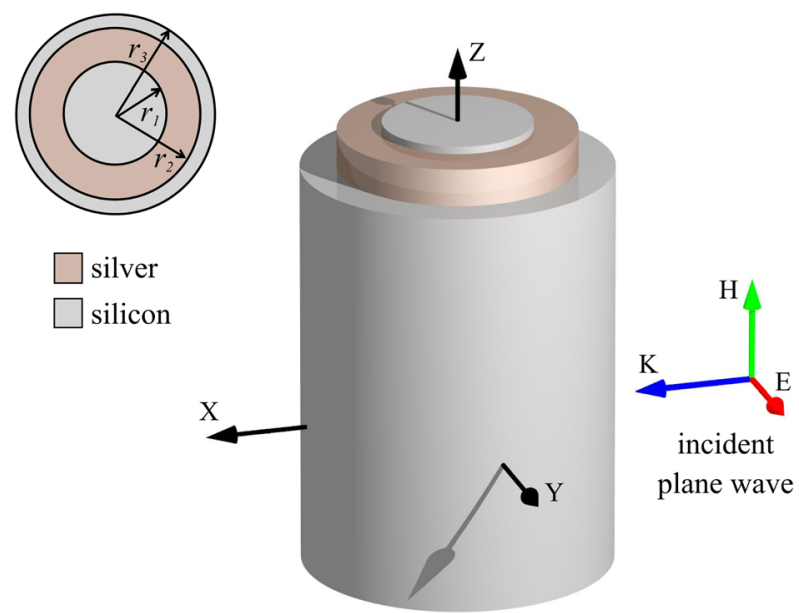

FIG. 1. Geometry of the problem: A three layer structure made of silicon and silver. The plane wave is illuminating from side with magnetic field parallel to the structure's axis. The structure is assumed to be relatively long compared to the wavelength, so it can be treated within a $2 \mathrm{D}$ problem. 
The total SCS for the structure can be expressed as a function of mode amplitudes as ${ }^{16} S C S=\frac{2 \lambda}{\pi} \sum_{n=0}^{+\infty} N(n)$ $\left|\rho_{L+1}^{n}\right|^{2}$, (taking into account $\left|\rho_{L+1}^{n}\right|=\left|\rho_{L+1}^{-n}\right|$ degeneracy $^{16,17}$ ). We normalize all SCS values to $2 \lambda / \pi$ introducing normalized SCS (NSCS).

The goal is maximizing the total SCS at an arbitrarily chosen wavelength by optimizing the number of layers and their parameters. Here, to simplify the problem, we choose particular materials, silver and silicon, as shown in Fig.1 and keep the thickness of the layers as the variables to be optimized. Here, we limit the outer radius of the structure to $87 \mathrm{~nm}$ to make the outcome comparable with previously published results of investigation superscattering effect in nanowires. ${ }^{5,14}$

As mentioned above, we choose silicon and silver to form the layers, and we use material data obtained from optical experiments. ${ }^{18} \mathrm{We}$ also notice that in small particles, whose size is comparable to the electron mean free path, the collision frequency of electrons is modified. This causes modification of the dielectric permittivity compared to bulk material. ${ }^{19}$ To take this effect into account, we use Drude model for describing silver, $\epsilon=\epsilon_{\infty}-\omega_{p}^{2} /\left(\omega^{2}+i \gamma \omega\right)$ where $\epsilon_{\infty}$ $=4.96^{18}$ and $\omega_{p}$ and $\gamma$ are plasma and bulk collision frequencies, respectively. First, for each frequency we use real $\left(\epsilon_{r}\right)$ and imaginary $\left(\epsilon_{i}\right)$ parts of the experimentally obtained dielectric permittivity ${ }^{18}$ to find $\gamma_{\text {bulk }}$ and $\omega_{p}$ by fitting the Drude formula $^{14} \gamma_{b u l k}=\omega \epsilon_{i} /\left(\epsilon_{\infty}-\epsilon_{r}\right)$ and $\omega_{p}^{2}=\omega^{2}\left[\left(\epsilon_{\infty}-\epsilon_{r}\right)^{2}+\epsilon_{i}^{2}\right] /$ $\left(\epsilon_{\infty}-\epsilon_{r}\right)$. Then, we update the value of collision frequency using $\gamma_{\text {small-particle }}=\gamma_{\text {bulk }}+A V_{f} / d$, where $A=1, V_{f}=1.388$ $\times 10^{6} \mathrm{~m} / \mathrm{s}$ (the Fermi velocity in silver) and $d$ is the characteristic size of the metallic structure. ${ }^{19} \mathrm{We}$ then substitute $\gamma_{\text {small-particle }}$ in Drude formula, and obtain $\epsilon$ which we use in our calculations.

To optimize the superscattering of the multilayer cylindrical structure for a desired wavelength, we use a GA to find the optimum set of the size parameters. GAs are used for finding a global extremum of a multi-dimensional target function within the defined parameter space. These adaptive algorithms have been employed in a verity of scientific and engineering problems forming computational models of "natural selection" and evolutionary systems. ${ }^{20-24}$ GAs are also used to optimize various electromagnetic properties of optical devices and systems ${ }^{25-32}$ and specifically scattering problems. ${ }^{33-38}$

Here, we briefly outline the algorithm that we use in our simulations. Figure 2 demonstrates the principles of a GA applied for our superscattering problem. To start, we form arrays of variables that we aim to optimize, which in our case are thicknesses of the layers. By analogy with definitions used in Gas, ${ }^{20-22}$ we call these sets of parameters as "chromosomes," while each variable within these chromosomes is a "gene." Figure 2(b) shows a chromosome for a 3layer structure. We generate large number (50 in our case) of random chromosomes, or individuals, for the first "generation" of optimization procedure. Next, we choose random pairs from these individuals to act as "parents," and we apply crossover and mutation processes to generate "offspring" for the next generation.

The crossover process involves braking parents' chromosomes and swapping their heads and tails between the (a)

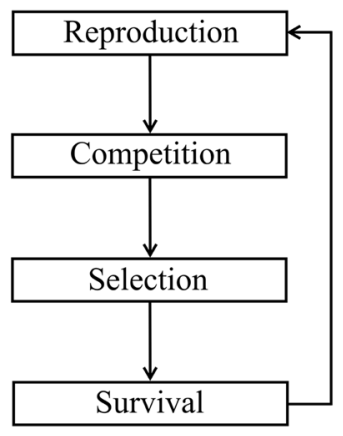

randomly chosen crossover points
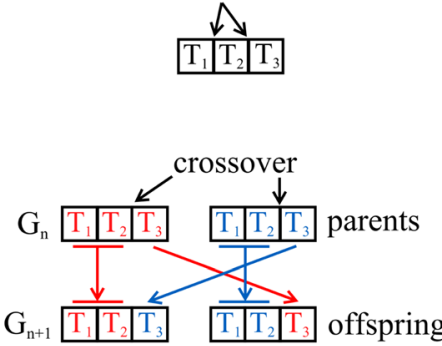

FIG. 2. (a) General flow of a genetic algorithm, and (b) crossover process applied to a three-layer structure. Chromosomes of parents are divided into two parts (head and tail) which are then swapped to generate offspring having their properties. The crossover point is chosen randomly. $T_{m}$ and $G_{n}$ indicate thickness of layer $m$ and generation $n$, respectively.

two chromosomes to form two new offspring. ${ }^{39}$ Figure 2(b) shows how crossover can be performed randomly at different points. Here, we use a one-point-crossover method, while it is also possible to use two-point-crossover to brake parents chromosomes into two points and swap only the middle genes for generating offspring. Mutation is the process of random change of a random gene to maintain "genetic diversity." 40 In our problem, mutation implies changing the value of a thickness of a layer within the defined radius range.

Next, we sort the individuals based on how close they are to our defined goal, which in our case is maximum scattering cross-section. Mathematically this means that we need "a fitness function" in order to evaluate each individual in the population. For superscattering problem, we define the following fitness function

$$
F_{i}\left(r_{1}, r_{2}, r_{3}\right)=\max \left\{N S C S\left(\lambda_{o p t}, r_{1}, r_{2}, r_{3}\right)\right\},
$$

where $i$ is the number of an individual, varying from 1 to the population number (50) and $\lambda_{\text {opt }}$ is the wavelength at which we want to optimize the SCS. The fitness function is used to evaluate each individual to assign fitness value. Then, a random quantity of the lowest fitness individuals is removed and they are replaced with the additionally generated offspring of the best parents. The best individuals are saved to enter next generations. When a better individual than the previous best one is generated, "Tabu search" technique is applied to find the local optimum value. ${ }^{41}$ This search technique can be applied for some random individuals in periodically chosen generations.

Another used technique is removing the population almost completely after several hundred or thousand generations and starting over by choosing new randomly generated individuals, similar to how it is done for the first generation. The difference is that in this step we use several previously found best individuals along with the new generation. This technique extends the coverage of the parameter space in order to find the global maximum fitness value.

Results of our optimization are shown in Fig. 3. This figure demonstrates that in optimal superscattering regimes, 

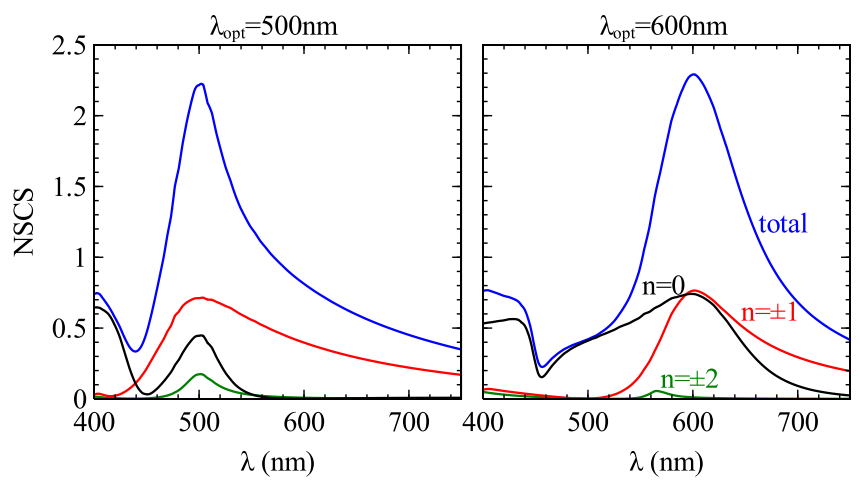

FIG. 3. Spectrum of the NSCS of optimized superscattering structures at different wavelengths. Shown is the total cross-section, as well as contributions of the first three modes.

several multipole resonances overlap at $\lambda_{\text {opt }}$. This effect is considerable in the case of $\lambda_{\text {opt }}=500 \mathrm{~nm}$, when the resonances of three different modes are overlapped. The contribution of the quadrupole mode becomes weaker for longer wavelength as it is shown in Fig. 3. This is caused by narrowing of the silver layer (see Table I for parameters). It can be also confirmed by comparing the amplitudes of the electric fields corresponding to different multipoles, presented in Fig. 4(a). According to this figure, the quadrupole mode has the largest amplitude at this particular wavelength, $\lambda_{\text {opt }}=500 \mathrm{~nm}$.

In Fig. 4(b), we show the far-field profiles for the structure which is optimized for $\lambda=500 \mathrm{~nm}$. Shown are field profiles at two different wavelength: $500 \mathrm{~nm}$ (superscattering) and $440 \mathrm{~nm}$ (weak scattering). The weak scattering regime is chosen such that SCS is minimal for our structure. Figure 4(b) clearly demonstrates that in the superscattering regime the particle creates large shadow behind the structure, and the shadow is much larger that the physical size of the particle.

It is also interesting to look at the far-field radiation pattern of the optimized structures. Figure 5 demonstrates the variation of the far-field radiation pattern with wavelength, for multi-layered structures optimized for different wavelengths. One can notice that at the optimal wavelength, the total scattering is maximal along with the directivity. Such relation between total scattering and directivity can be understood by using the optical theorem, according to which the overall extinction is proportional to the forward scattering. Using our parameters, the scattering dominates the absorption. ${ }^{42}$ Stronger scattering leads to better directivity, making our optimized multi-layered structures good candidates for subwavelength optical nanoantennas.

TABLE I. Results of the superscattering optimization for a three-layer plasmonic structure (see Fig. 1) for several wavelengths. All dimensions are in $\mathrm{nm}$.

\begin{tabular}{lllll}
\hline \hline$\lambda_{\text {opt }}$ & $r_{1}$ & $r_{2}$ & $r_{3}$ & $N S C S_{\max }$ \\
\hline 450 & 43 & 83 & 87 & 1.909 \\
500 & 49 & 78 & 87 & 2.211 \\
550 & 49 & 72 & 87 & 2.261 \\
600 & 47 & 66 & 87 & 2.290 \\
650 & 36 & 58 & 87 & 2.287 \\
\hline \hline
\end{tabular}
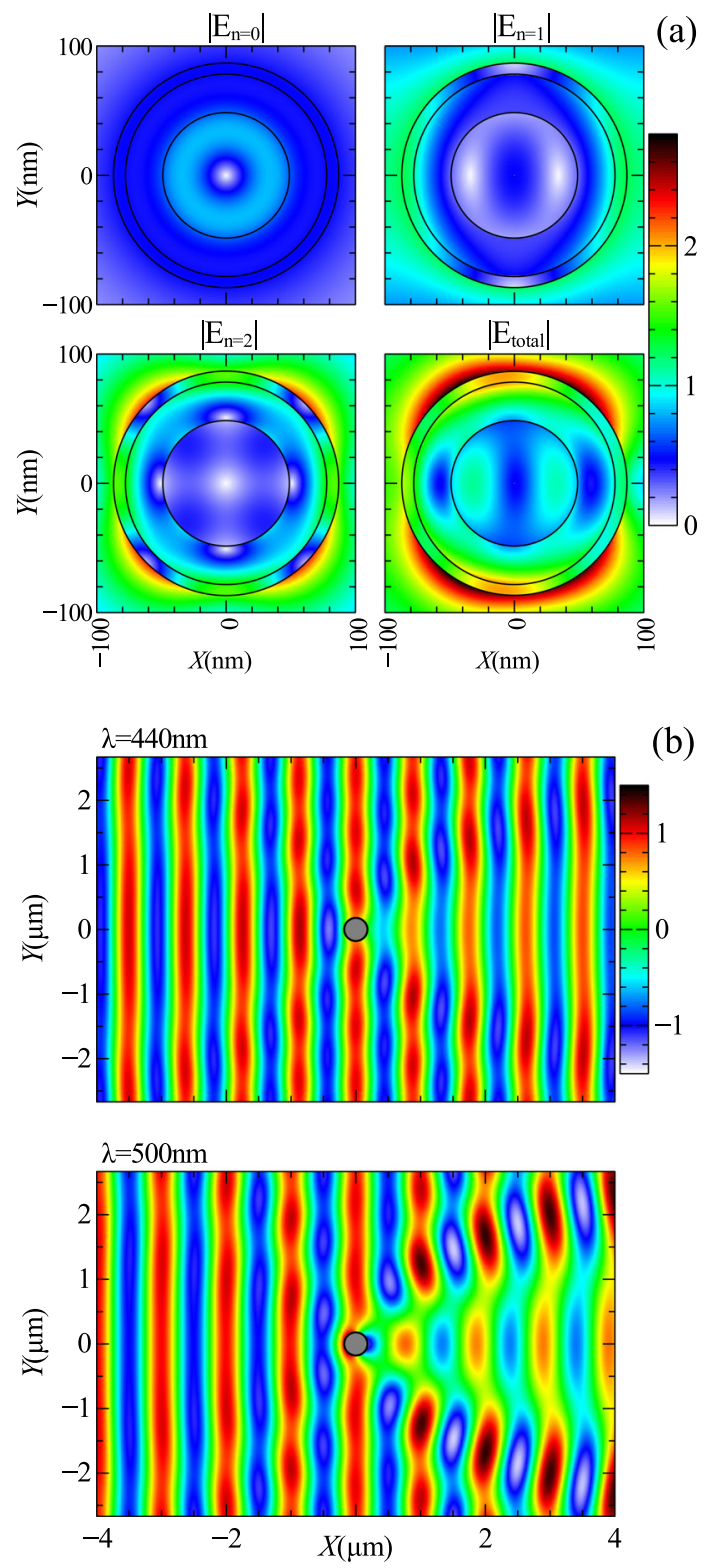

FIG. 4. Field profile of the optimized structure at $\lambda=\lambda_{\text {opt }}=500 \mathrm{~nm}$. (a) Electric field profile for the first three modes as well as the total electric field. (b) The far field distribution for at two different wavelengths, $440 \mathrm{~nm}$ and $500 \mathrm{~nm}$ showing weak scattering and super scattering regimes. The plane wave is incident from left and the final field profile is calculated using first ten modes within the structure.

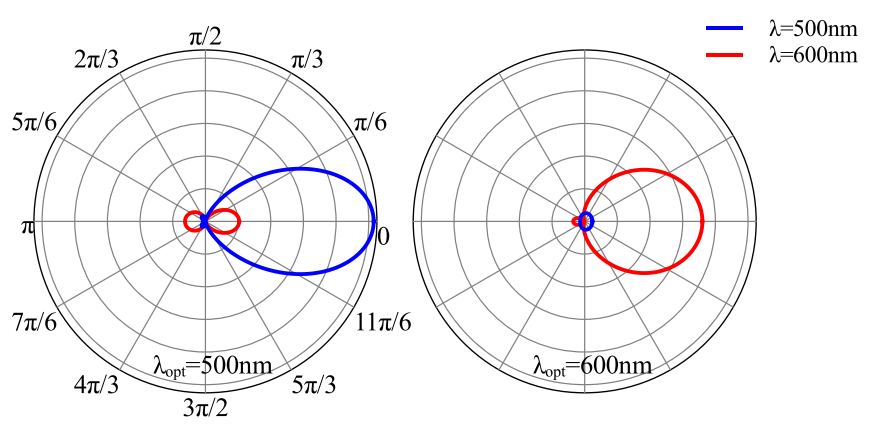

FIG. 5. Far field radiation pattern of structures optimized for different wavelengths. The plane wave is incident from left. Both panels have the same radial tick steps. 
Table I summarizes the parameters of several optimized structures. The results show that the shift of superscattering spectrum is mostly controlled by tuning the outer radius of the silver layer $r_{2}$. This can intuitively be understood from the total field profile shown in Fig. 4(a). Indeed, we observe that the field amplitude is the largest near the outer layer, and if we assume that the overall size of the structure is constant (see Table I), then variation of outer radius of metallic layer $r_{2}$, will affect the field stronger than if we change the radius of the internal silicon layer $r_{1}$.

In conclusion, we have demonstrated that a genetic algorithm can be employed for optimizing superscattering phenomena. Using material parameters obtained in experiments, we designed core-shell nanostructures which by spectrally overlapping several resonant modes, show the superscattering effect at any desired wavelengths. In general, this method will also be efficient for optimizing cloaking properties and other scattering characteristics of core-shell nanowires and other nanoparticles.

The authors acknowledge a support from the Australian Research Council through the Future Fellowship (FT110100037) and the Discovery Project programs and also thank S. Fan and W. Liu for their interest in this work.

${ }^{1}$ N. Zheludev and Y. Kivshar, Nat. Mater. 11, 917-924 (2012).

${ }^{2}$ A. Alu and N. Engheta, Phys. Rev. Lett. 100, 113901 (2008).

${ }^{3}$ D. Filonov, A. Slobozhanyuk, P. Belov, and Y. Kivshar, Phys. Status Solidi RRL 6, 46 (2012).

${ }^{4}$ P. Chen, J. Soric, and A. Alù, Adv. Mater. 24, OP281 (2012).

${ }^{5}$ Z. Ruan and S. Fan, Phys. Rev. Lett. 105, 013901 (2010).

${ }^{6}$ H. Atwater and A. Polman, Nat. Mater. 9, 205-213 (2010).

${ }^{7}$ S. Pillai, K. Catchpole, T. Trupke, and M. Green, J. Appl. Phys. 101, 093105 (2007).

${ }^{8}$ F. Hao, Y. Sonnefraud, P. Dorpe, S. Maier, N. Halas, and P. Nordlander, Nano Lett. 8, 3983-3988 (2008).

${ }^{9}$ K. Gracie, E. Correa, S. Mabbott, J. Dougan, D. Graham, R. Goodacre, and K. Faulds, Chem. Sci. 5, 1030 (2014).

${ }^{10}$ W. Qian, X. Huang, B. Kang, and M. El-Sayed, J. Biomed. Opt. 15, 046025 (2010).

${ }^{11}$ L. Shang, H. Chen, L. Deng, and S. Dong, Biosens. Bioelectron. 23, 1180-1184 (2008).

${ }^{12}$ Z. Ruan and S. Fan, Appl. Phys. Lett. 98, 043101 (2011).
${ }^{13}$ L. Verslegers, Z. Yu, Z. Ruan, P. Catrysse, and S. Fan, Phys. Rev. Lett. 108, 083902 (2012).

${ }^{14}$ A. Mirzaei, I. Shadrivov, A. Miroshnichenko, and Y. Kivshar, Opt. Express 21, 10454 (2013).

${ }^{15}$ L. Schchter, Beam-Wave Interaction in Periodic and Quasi-Periodic Structures (Springer, 2011).

${ }^{16}$ C. A. Balanis, Advanced Engineering Electromagnetics (Wiley, 1989).

${ }^{17}$ M. I. Tribelsky and B. S. Lukyanchuk, Phys. Rev. Lett. 97, 263902 (2006).

${ }^{18}$ E. Palik, Handbook of Optical Constants of Solids (Academic Press, 1997).

${ }^{19}$ U. Kreibig, J. Phys. F: Metal Phys. 4, 999-1014 (1974).

${ }^{20} \mathrm{M}$. Mitchell, An Introduction to Genetic Algorithms (MIT Press, 1998).

${ }^{21} \mathrm{G}$. Winter, Genetic Algorithms in Engineering and Computer Science (John Wiley, 1995).

${ }^{22}$ R. Haupt and S. Haupt, Practical Genetic Algorithms (John Wiley, 2004).

${ }^{23}$ D. E. Goldberg, Genetic Algorithms in Search, Optimization, and Machine Learning (Addison-Wesley, 1989).

${ }^{24}$ D. Whitley, Stat. Comput. 4, 65 (1994).

${ }^{25}$ Y. Rahmat-Samii and E. Michielssen, Electromagnetic Optimization by Genetic Algorithms (John Wiley, 1999).

${ }^{26} \mathrm{R}$. Haupt and D. Werner, Genetic Algorithms in Electromagnetics (John Wiley, 2007).

${ }^{27}$ L. Sanchis, A. Hkansson, D. Lpez-Zann, J. Bravo-Abad, and J. SnchezDehesa, Appl. Phys. Lett. 84, 4460 (2004).

${ }^{28}$ M. dAvezac, J. Luo, T. Chanier, and A. Zunger, Phys. Rev. Lett. 108, 027401 (2012).

${ }^{29}$ Md. T. Arafin, N. Islam, S. Roy, and S. Islam, Opt. Quantum Electron. 44, 701-715 (2012).

${ }^{30}$ F. Omenetto, B. Luce, and A. Taylor, J. Opt. Soc. Am. B 16, 2005 (1999).

${ }^{31}$ S. Singh, S. Saini, G. Kaur, and R. Kaler, Opt. Eng. 53, 016103 (2014).

${ }^{32}$ W. Zhang, C. Wang, J. Shu, C. Jiang, and W. Hu, IEEE Photonics Technol. Lett. 16, 1652 (2004).

${ }^{33}$ Y. Fan, T. Jiang, and D. Evans, Int. J. Comput. Math. 79, 573 (2002).

${ }^{34}$ Z. Yu, Y. Feng, X. Xu, J. Zhao, and T. Jiang, J. Phys. D: Appl. Phys. 44, 185102 (2011).

${ }^{35}$ W. Chien, Prog. Electromagn. Res. 82, 1 (2008).

${ }^{36}$ G. Gazonas, D. Weile, R. Wildman, and A. Mohan, Int. J. Solids Struct. 43, 5851-5866 (2006).

${ }^{37}$ X. Wang and E. Semouchkina, Appl. Phys. Lett. 102, 113506 (2013).

${ }^{38}$ X. Wang, F. Chen, and E. Semouchkina, AIP Adv. 3, 112111 (2013).

${ }^{39}$ T. Gwiazda, Genetic Algorithms Reference Vol.1 Crossover for SingleObjective Numerical Optimization Problems (Lightning Source, Inc., 2006).

${ }^{40} \mathrm{~K}$. Man, K. Tang, and S. Kwong, Genetic Algorithms: Concepts and Designs (Springer, 1999).

${ }^{41}$ F. Glover, Comput. Oper. Res. 13, 533-549 (1986).

${ }^{42} \mathrm{C}$. Bohren and D. Huffman, Absorption and Scattering of Light by Small Particles (John Wiley, 1983). 